\title{
Current commentaries about deep dyspareunia and the genito-urinary syndrome of menopause
}

\begin{abstract}
The authors carried out an evaluation of the genito-urinary syndrome of menopause, which is defined as a set of signs and symptoms associated with the reduction of estrogen and other sex steroids involving changes in labia maiora and minora, clitoris, vaginal vestibule, urethra and bladder, which create discomfort and are not better explained by another diagnosis. The syndrome may include, but not be limited to, genital symptoms of dryness, burning and irritation, sexual symptoms of lack of lubrication, discomfort or pain, and impaired functions, and may be associated with urinary symptoms of urgency, dysuria and recurrent urinary infections. Affected women may have some or all these signs and symptoms; however, specifically regarding deep dyspareunia, a discomfort caused by pain felt in the vaginal fundus during sexual relations, a better explanation is the relative short vagina syndrome. This syndrome is related to incompatibility of penis size with the size of the extended vagina or to an organic cause such as loss of vaginal elasticity due to atrophy caused by the lack of sex steroids, with consequent vaginal shortening and reduced capacity of vaginal distention. Accepting this explanation facilitates the approach to this symptom and permits gynecologists to treat affected women in an appropriate manner. The treatment of this type of discomfort requires changes in sexual practices during the climacteric and menopause, together with hormone replacement, as recommended by professionals who are aware of the two syndromes.
\end{abstract}

Volume 5 Issue 3- 2017

\author{
Matthes ACS,' Zucca Matthes G' \\ 'Professor and Coordinator of Gynecology \& Obstetrics, \\ Medical School of University of Ribeirão Preto, Brazil \\ ${ }^{2}$ Ph.D Professor, Medical School of Botucatu, UNESP, Brazil \\ Correspondence: Ângelo CS Matthes, Ph.D Professor, Medical \\ School of Botucatu, UNESP, Mastologist, Cancer Hospital of \\ Barretos, Av Costabile Romano, 220, Ribeirão Preto, 14096-000, \\ Brazil,Tel 55 (I6) 3603-7000/55 (16) 981 4585 65, \\ Email amatthes@unaerp.br
}

Received: October 17, 2016 | Published:June 22, 2017

\section{Introduction}

Deep dyspareunia due to vaginal atrophy should be treated with hormone replacement and changes in sexual practices that will avoid this discomfort. In May 2013, the International Society for the Study of Women's Sexual Health (ISSWSH) and the North American Menopause Society (NAMS) co-sponsored a consensus conference which established the current terminology for genito-urinary tract symptoms related to menopause defined as Genito-Urinary Syndrome of Menopause (GSM), ${ }^{1}$ which includes sexual symptoms such as lack of lubrication, discomfort or pain and impaired function in addition to genital and urinary symptoms. The basis used by these societies for the inclusion of all these symptoms in GSM was hypoestrogenism, a condition also commonly found in women with premature ovarian failure who have amenorrhea or are postmenopausal, as well breastfeeding women and others with hyperprolactinemia and women taking gonadotropin-releasing hormone analogues $(\mathrm{GnRH})$ for the treatment of endometriosis and of estrogen receptor-positive tumors. ${ }^{2}$ Indeed many menopausal women may present some or all the sexual symptoms involved in GSM. However, specifically the cause of the pain in the vaginal fundus felt during the sex act, called deep dyspareunia (DD), is not the lack of sex hormones, but rather another external variable, i.e., the size of the penis of the partner which is incompatible with the size of the extended vagina. Or there may be an organic cause in the vaginal fundus which causes pain with the simple touch of the penis. On the other hand, many women with GSM who are sexually inactive with no vaginal penetration and who have marked hypoestrogenism do not suffer DD, since this symptom is always associated with sexual penetration by a penis of a size that is not compatible with the size of the vagina, The vaginal atrophy detected in these women does not cause local pain per se, with pain occurring only, for example, during gynecological examination.

In contrast, women with hypoestrogenism who are sexually active with vaginal penetration may develop relative short vagina syndrome $(\text { RSVS })^{3}$ since vaginal atrophy is a determinant factor causing the vagina to be less elastic and less flexible. Even women of any age with a common complaint of DD, with normal or reduced sex steroid levels or even higher than expected levels, may have pelvic pain after repeated or traumatic sexual relations. When this condition is maintained or even before the occurrence of low abdominal pain, these women start to have sexual complaints such as loss of libido and sexual aversion, using excuses to reduce or even avoid intercourse. As a consequence, the marital relationship is negatively affected, with mutual complaints possibly leading to the end of the marital relationship.. The cause of the sexual problem may be attributed to the woman, who will then develop a low self-esteem. Affected women may develop stress and/ or depression or anxiety and may modify their eating habits, gaining or losing weight. This set of symptoms associated with some signals represent the RSVS. ${ }^{3}$ Many women with genito-urinary syndrome of menopause (GSM) or undergoing treatment for breast cancer at any age will never develop RSVS despite having low sex steroid levels. ${ }^{4}$

An atrophic vagina due to hypoestrogenism and/or radiotherapy is an organic cause of dyspareunia. ${ }^{5-7}$ Some couples do not show incompatibility between penis size and the vagina while the woman maintains good vaginal trophism. However, incompatibility may occur during menopause or after some years of antiestrogen use, after radiotherapy or after some time of absence of sexual activity with penetration. Thus, the women start to have pain in the vaginal fundus 
during intercourse accompanied by chronic pelvic pain (CPP). ${ }^{5} \mathrm{CPP}$ is highly prevalent and also debilitating, with a marked impact on quality of life and productivity, as well as significant costs for health services. ${ }^{5}$ Women are known to have more abdominal pain than men, ${ }^{8}$ a plausible explanation for this fact being that they are penetrated and subjected to penis-caused traumatism during intercourse. One third of male to female transexuals under estradiol and/or antiandrogen treatment develop chronic pain, ${ }^{8}$ an occurrence obviously explained by the fact that, by taking hormonal estrogen medication, they become more feminized and more sexually active with penetration of the constructed vagina. This involves a greater chance of trauma to the vaginal dome and pelvic pain when there is incompatibility between penis size and size of the distended vagina. ${ }^{9}$

Curran ${ }^{8}$ stated that "Before a woman has entered a pain clinic, however, she usually sees a gynecologist. You may decide to treat empirically or find at laparoscopy that your suspicions are confirmedshe has endometriosis, which you treat. Initially the pain gets better but she returns some months later with recurrence of pain. This is most likely because of central sensitization and pain memory, akin to how patients develop phantom limb pain. You have treated the causethe surgery was successful-so she might be keen to have another operation thinking there must be more endometriosis causing the problem. If you decide to operate and find nothing, this can leave the clinician and patient wondering why she still has pain. Chronic pain mechanisms explain why this is the case -pain has become the condition and she might benefit from seeing a specialist with a simple explanation of chronic pain to set up this collaboration rather than any suggestion that the symptoms are not medically explainable or have "no organic cause". This is the point: it is necessary to talk about the incompatibility between penis and vagina, to determine the size of the penis, to find out which sexual positions cause the most pain, and to instruct the couple to change their sexual practices in order to avoid deep penetration because, in contrast to what the cited author states "Initially the pain gets better but she returns some months later with recurrence of pain.....and she might benefit from seeing a specialist with a simple explanation of chronic pain to set up this collaboration rather than any suggestion that the symptoms are not medically explainable or have "no organic cause", ${ }^{8}$ a plausible explanation is that, when a woman is under investigation by a gynecologist or is being submitted to exams such as laparoscopy, the couple tends to have fewer sex relations.

Thus, the pain first improves and later returns with the resumption of sexual activity, and since the women have no convincing explanations they end up resigning themselves to the pain or continue to look for further medical consultation. Indeed, we receive women who have already consulted with more than five professionals without solving their problem and who continue to have a poor quality of life. Women with GSM plus RSVS may improve their quality of life with hormone replacement therapy and changes in sexual attitudes. Sexual activity should be constant and progressive in order to obtain gradual vaginal adaptation. ${ }^{9}$ Ideally, women who do not have RSVS should be instructed about their vaginal size and the penis size of their partners so that they may adopt positions that would prevent traumatic penetration of the vagina. ${ }^{10}$ However, women who already have RSVS, if they have a parametrial inflammation ${ }^{11}$ should observe sexual rest for 20days after the diagnosis, taking anti-inflammatory and local or systemic hormonal drugs during this period depending on hormonal indication, or opt for other treatments including the use of opemifene, microablative fractional laser sessions, and dehydroepiandrosterone. ${ }^{5}$
After this period, the couples should be instructed to consider a change in sexual practice, with manipulation of the clitoris and little or no vaginal penetration, or with progressive and constant penetration for several days until deep vaginal penetration can be performed without pain. However, it should be remembered that medications are not sufficient and that sexual behavior should be changed since dyspareunia is the pain that arises only during intercourse.

The relief of the qualitative parameter, DD, is not directly caused by the action of medications, but rather represents a response to this action on the loss of elastic rugosity of the vagina during menopause, which is influenced by several variables such as age, race, sex, repeated sexual movements, tissue injuries and other factors, all of them depending on the disaggregation of the collagen that supports the vaginal epithelium. ${ }^{12}$ The variable that only concerns DD is the capacity of vaginal distention, which depends on vaginal elasticity or on the presence or absence of an organic cause such as an atrophic vagina, which is an organic cause of $\mathrm{DD}$ in menopausal women who are not on hormone replacement therapy.

The ideal would be for a woman to be aware of the capacity of distention of her vagina in order to ask her partner to adopt positions that will prevent a penetration exceeding the capacity of distention of her vagina. ${ }^{13}$ Several studies have shown the clinical relevance of a short vagina as an organic cause of deep dyspareunia. ${ }^{11,14-19}$ and Binik $^{20}$ has correctly stated that different dyspareunia syndromes are likely to exist. RSVS is one of them and therefore a new DD concept should be introduced in the medical literature for the understanding of this syndrome, which always starts with DD. First of all, DD pain only occurs during intercourse. The pain a woman feels before and after coitus should be considered to be genital pain and not dyspareunia. The concept that dyspareunia includes genital pain immediately before, during and after coitus should be abandoned. Thus, deep dyspareunia is defined as the pain a woman feels in the vaginal fundus during coitus, superficial dyspareunia is defined as the pain a woman feels in the vulva and in the vaginal introitus during intercourse, and male sex dyspareunia is defined as the pain a man feels in his penis or pelvis during intercourse. Thus, dyspareunia is exclusively the pain felt during intercourse. Second, the pain felt in the vaginal opening (superficial dyspareunia) should be distinguished from DD. There are reports of pain when the penis touches the vaginal opening or of pain only in the vaginal fundus, or both types of pain may be present. Exclusive superficial dyspareunia does not cause RSVS.

Third, new concepts should be introduced:

i. Primary dyspareunia, which should be defined as pain during coitus without an organic cause; pain occurs because of incompatibility between penis size and vaginal size, which undergoes distention beyond its limit of flexibility.

ii. Secondary dyspareunia due to an organic cause such as endometriosis or some other condition, so that when the penis touches the affected tissue the woman feels pain. The concept that primary dyspareunia is the first occurrence of pain and secondary dyspareunia is the recurrence of pain after a pain-free period should be abandoned since pain during intercourse is always related to the penetration of the vagina by the penis. To illustrate, if a widow who had dyspareunia in her first marriage and had no sexual activity for five years and therefore no DD, marries again and still feels pain during intercourse, she should be diagnosed as having primary dyspareunia if the only cause of pain is incompatibility 
between penis and vagina, and as having secondary dyspareunia if an organic cause has now arisen, such as vaginal atrophy or another.

iii. Finally, there is a limit to the capacity of vaginal distention. The concept that during orgasm the vagina grows more than double in size in order to accommodate the penis is incorrect and should also be abandoned. The vagina only distends $3.0 \mathrm{~cm}(2.5 \mathrm{~cm}$ more or less), as determined with the use of the vaginal meter of Matthes. ${ }^{13}$

On this basis, the objective of the present article was to comment about GSM in order to show all doctors in general, and especially gynecologists, that DD, one of its symptoms, is not caused by hypoestrogenism but rather by a penis that penetrates an atrophic vagina. This may cause injury to the vaginal fundus, with the consequent occurrence of RSVS, which is another diagnosis that better explains this sexual symptom, also related to GSM. Thus, it is important for all gynecologists to be aware of RSVS in order to counsel and treat their patients so that they might experience a sexual life of better quality. On this basis, the understanding of RSVS opens a very extensive research field and more studies should be conducted to expand our knowledge of the size and maximum flexibility of the vagina, as in the case, for example, of women before and after hysterectomy. A longitudinal study is also necessary in order to understand the changes in vaginal flexibility that occurs during the life of a woman, as well as racial variations. Such study would provide significant benefits for the $30 \%$ group of women who experience DD and CPP with a reduction of sexual pleasure, although more studies are needed in order to investigate RSVS.

\section{Acknowledgements}

We are grateful to Prof. Reinaldo Bestetti, Coordinator of the Medical Course of the University of Ribeirão Preto.

\section{Conflict of interest}

The author declares no conflict of interest.

\section{References}

1. Portman DJ, Gass MLS. Genitourinary syndrome of menopause: new terminology for vulvovaginal atrophy from the International Society for the Study of Women's sexual health and the North American menopause society. Menopause. 2014;21(10):1063-1068.

2. Matthes ACS, ZuccaMatthes G, Oliveira MA. The genito urinary syndrome of menopause presents sexual symptoms that can be best explained by the relative short vagina syndrome. Gynecol Obstet. 2016;6:382.

3. Matthes ACS, Zucca-Matthes G. Description of a medical condition: RSVS - Relative short vagina syndrome. RBSH. 2012;23:31-38.

4. Matthes ACS, Zucca-Matthes G. Letters do the Editor. Dyspareunia an ever-present discussion. Menopause. 2016;23:698.

5. Bachmann G, Phillips N. Dyspareunia: including this diagnosis in chronic pain reporting. Menopause. 2016;23(1):1-2.
6. Stinesen Kollberg K, Waldenström AC, Bergmark K, et al. Reduced vaginal elasticity, reduced lubrication, and deep and superficial dyspareunia in irradiated gynecological cancer survivors. Acta Oncol. 2015;54(5):772779 .

7. Bregendahl S, Emmertsen KJ, Lindegaard JC, et al. Urinary and sexual dysfunction in women after resection with and without preoperative radiotherapy for rectal cancer: a population-based cross-sectional study. Colorectal Dis. 2015;17(1):26-37.

8. Curran NC. Commentary on the influence of gender on the management of chronic pelvic pain. Br J Obstet Gynaecol. 2015;122(6):766-768.

9. Matthes ACS, Matthes GZ. A comment about the quantitative analysis of normal vaginal shape and dimension. Int Urogynecol J. 2016;27(10):16091610 .

10. Hunter MN, Nakagawa S, Van Den Eeden SK, et al. Predictors of impact of vaginal symptoms in postmenopausal women. Menopause. 2016;23(1):4046.

11. Nascu PC, Vilos GA, Ettler HC, et al. Histopathologic findings on uterosacral ligaments in women with chronic pelvic pain and visually normal pelvis at laparoscopy. J Minim Invasive Gynecol. 2006;13(3):201204.

12. Tinelli A, Malvasi A, Rahimi S, et al. Age-related pelvic floor modifications and prolapse risk factors in postmenopausal women. Menopause. 2010;17(1):204-212.

13. Matthes ACS, Zucca-Matthes G. Measurement of vaginal flexibility and its involvement in the sexual health of women. $J$ Women's Health Care. 2016;5:302-305

14. Phillips J. Dyspareunia and its surgical treatment. $\mathrm{Br}$ Med $\mathrm{J}$. 1914;1(2788):1232-1234.

15. Kompanje EJ. Painful sexual intercourse caused by a disproportionately long penis: an historical note on a remarkable treatment devised by Guilhelmius Fabricius Hildanus (1560-1634). Arch Sex Behav. 2006;35(5):603-605.

16. Abdelmonem AM. Vaginal length and incidence of dyspareunia after total abdominal versus vaginal hysterectomy. Eur J Obstet Gynecol Reprod Biol. 2010;151(2):190-192.

17. Sibert L, Safsaf A, Rigaud J, et al. Pelvic sexual pain. Prog Urol. 2010;20(12):967-972.

18. Moshesh M, Olshan AF, Saldana T, et al. Examining the relationship between uterine fibroids and dyspareunia among premenopausal women in the United States. J Sex Med. 2014;11(3):800-808.

19. Fritzer N, Tammaa A, Haas D, et al. When sex is not on fire: a prospective multicentre study evaluating the short-term effects of radical resection of endometriosis on quality of sex life and dyspareunia. Eur J Obstet Gynecol Reprod Biol. 2016;197:36-40.

20. Binik YM. The DSM diagnostic criteria for dyspareunia. Arch Sex Behav. 2010;39(2):292-303. 